\title{
Machine Learning for Predictive Maintenance with Smart Maintenance Simulator
}

\author{
Abdessalam Lmouatassime \\ LTI Laboratory, National School of Applied Sciences \\ Chouaib Doukkali University \\ El Jadida, Morocco
}

\begin{abstract}
Machine learning is a vital part of today's world. In Industry 4.0, Machine Learning approach for Predictive Maintenance continues to generate research attention, especially with the AI implementation. Indeed, the benefits of this approach such as helping determine the condition of equipment and predicting when maintenance should be performed, are extremely strategic. In this article, we propose a new Sensors Reference Model (SRM) and architecture for a Smart Maintenance Simulator (SmaSim) based on a new advanced connected sensors called Smart Sensors. This SmaSim is a low code simulator which can help researchers, engineers, and practitioners to select appropriate Smart Sensors and Machine Learning algorithms for predictive maintenance in Smart Factories.
\end{abstract}

\section{Keywords}

Industry 4.0; Predictive maintenance; Machine Learning; Smart Maintenance Simulator; Smart Sensors

\section{INTRODUCTION}

Industry 4.0 is revolutionizing the way companies manufacture, improve and distribute their products. Manufacturers are integrating enabling approaches, including predictive maintenance and machine learning. These smart factories are equipped with advanced connected sensors called Internet of Things (IoT) that collect data and by applying machine learning, manufacturers can predict and detect errors, rather than at later stages when repair work is more expensive. But what is industry 4.0? what is predictive maintenance? And what is Machine Learning?

\subsection{What is Industry 4.0?}

The term Industry 4.0 was coined in Germany in 2011 , this term refers to the introduction of internet-connected technologies (meaning internet protocols and software methodology as well as software, sensors, and actuators connected to networks) within an industrial context [1].

The industrial revolution led to changes in the labor market with machines replacing human labor. As shown on the figure 1, the first industrial revolution replaced manual work with the invention of a steam engine and the second industrial revolution enabled mass production using electric energy [2]. The tertiary industrial revolution started the automation era with informatization based on computers and the Internet [3]. In the future, the super intelligence revolution based on the Internet of things, cyber-physical system, and artificial intelligence (AI) will greatly change human intellectual labor [4].

\author{
Mohammed Bousmah \\ LTI Laboratory, National School of Applied Sciences \\ Chouaib Doukkali University \\ El Jadida, Morocco
}

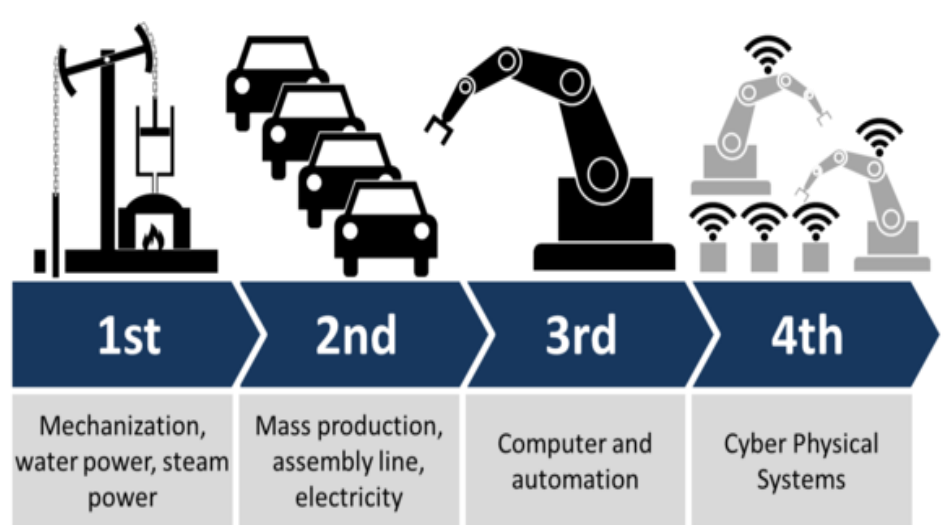

Fig. 1 The four industrial revolutions (Wikipedia)

When industry 4.0 is correctly implemented, the combination of the increased availability of sophisticated sensors with the transmission capacity available via the internet of things (IoT), backed by increasing computational power, will enable a new level of data resolution and new ways to synthesize and analyse data that companies can use to improve many areas of operations [5]. The result will be responsive and agile production processes that ensure and enhance performance across a range of industrial sectors.

\subsection{What is predictive maintenance?}

Techniques for maintenance policies can be categorized into the following main classifications:

- Corrective Maintenance $(\mathrm{CrM})$ : It is a reactive maintenance technique which is performed only when the equipment has failed.

- Preventive Maintenance (PvM): It is a time-based maintenance which refers to periodically performed maintenance based on a planned schedule in order to anticipate the failures.

- Predictive Maintenance (PdM): it allows failure detection at an early stage based on engineering techniques such as machine learning approaches.

The main objective of PdM based on ML approaches is to minimize equipment failure rates, improve equipment condition, prolong the life of the equipment, and reduce the maintenance costs.

In predictive maintenance approach, data is collected over time to monitor the state of equipment. The goal is to find patterns that can help predict and ultimately prevent failures. Predictive maintenance is a subject to many recent research papers with the long history behind it. It refers to the intelligent monitoring of equipment to avoid future failures. Predictive maintenance has evolved from the first method that is visual inspection to automated methods using the advanced signal processing techniques based on pattern recognition and machine learning, 
neural networks, fuzzy logic, etc. [6]. The automated methods provide viable solution to many industries detecting and collecting sensitive information from the equipment. Together with integrated sensors, predictive maintenance can avoid unnecessary equipment replacement, reduce machine downtime, find the root cause the fault, and in this way save costs and improve efficiency [7]. Predictive maintenance overlaps with the scope of preventive maintenance in terms of scheduling the maintenance activity in advance to avoid machine failures. In contrast to conventional preventive maintenance, predictive maintenance schedules activities are based on collected data from sensors and analysis algorithms [8].

\subsection{What is Machine Learning?}

According to Samuel, A.L. [9], Machine Learning is the "Field of study that gives computers the ability to learn without being explicitly programmed".

Machine learning is a branch of artificial intelligence (AI) focused on building applications that learn from data and improve their accuracy over time without being programmed. In traditional programming approach, answers and decision are made from data and a sequence of program instruction called rules. As shown on the figure 2 below, in Machine Learning approach, machines are 'trained' to find rules, patterns and features from massive amounts of data and answers, in order to make decisions and predictions based on new data. The better the algorithm, the more accurate the decisions and predictions will become as it processes more data.
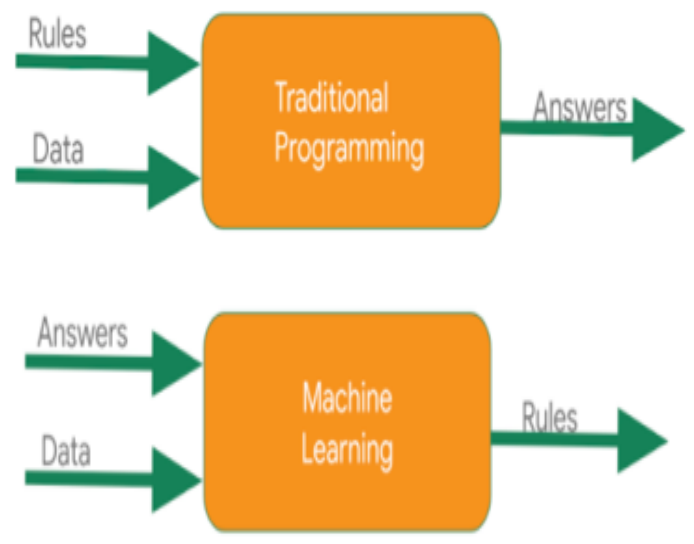

Fig. 2 Traditional programming vs Machine Learning es
approach

Machine learning algorithms are often categorized as supervised, unsupervised or reinforcement learning.

Supervised machine learning algorithms can apply what has been learned in the past to new data using labelled examples to predict future events. Starting from the analysis of a known training dataset, the learning algorithm produces an inferred function to make predictions about the output values. The system is able to provide targets for any new input after sufficient training. The learning algorithm can also compare its output with the correct, intended output and find errors in order to modify the model accordingly.

In contrast, unsupervised machine learning algorithms are used when the information used to train is neither classified nor labeled. Unsupervised learning studies how systems can infer a function to describe a hidden structure from unlabeled data. The system doesn't figure out the right output, but it explores the data and can draw inferences from datasets to describe hidden structures from unlabeled data.
Reinforcement machine learning algorithms is a learning method that interacts with its environment by producing actions and discovers errors or rewards. Trial and error search and delayed reward are the most relevant characteristics of reinforcement learning. This method allows machines and software agents to automatically determine the ideal behavior within a specific context in order to maximize its performance. Simple reward feedback is required for the agent to learn which action is best; this is known as the reinforcement signal.

There are four basic steps for building a machine learning application (or model).

\section{Step 1: Prepare a training Dataset \\ Step 2: Create a model \\ Step 3: Train the model \\ Step 4: Test and deploy the model}

In this article, we propose an example of machine learning for predictive maintenance in Smart Factories.

\section{RELATED WORKS}

This study aims to present a comprehensive literature review to discover existing studies and ML applications in Predictive Maintenance.

J. Dalzochio, R. Kunst, E. Pignaton et al. article [10] give a detailed literature on the machine learning applied to PdM. AI and ML techniques are used in different phases of PDM and different purpose like classification, prediction, and validation of the retained models.

Researchers tend to test multiple machine learning techniques to find the best one that deals with their data and their specific problems: fault prediction, Anomaly detection, RUL estimation, Failure prediction, time series prediction, different Classifications.

Among them we find a variety of techniques very well mind mapped in figure 3 :

- ANN : Artificial neural network

- $\quad$ LSTM : Long- Short Term Memory

- GRU: Gated Recurrent Unit

- FFNN: Feed Forward Neural Network

- MLP : Multilayer Perceptron

- $\quad$ BPNN : Back-propagation Neural network

- $\quad$ ENN : Elman Neural Network

- $\quad$ RBNN : Radial Basis Function Neural network

- PNN: Probabilistic Neural Network

- $\quad$ FNN : Fuzzy neural network

- WNN : Wavelet neural network

- $\quad \mathrm{KNN}$ : K-Nearest Neighbors

- DT : Decision Trees

- RF : Random Forests

- NB : Naive Bayes

- SVM : Support Vector machine

- BN: Bayesian network

- DBN : Dynamics Bayesian Network

- ARMA : AutoRegressive moving-average

- ARIMA: AutoRegressive Integrated Moving Average

- GBM : Gradient boosting machine

- DRF : Distributed Random Forest

- XGBoost : Extreeme gradiant boosting

- CNN: Convolutional neural network

- DNN: Deep Neural Network

- DTL : Decision tree learning 
Table 1. Machine learning techniques and sensors used in PdM

\begin{tabular}{|c|c|c|c|c|c|c|c|c|c|c|c|c|c|c|c|c|c|c|c|c|c|c|c|c|c|c|}
\hline \multirow[t]{2}{*}{ Ref } & \multicolumn{8}{|c|}{ Sensors } & \multicolumn{18}{|c|}{ Technologies used } \\
\hline & sound & id limage & vibration & Temperature & pressure & flow & electric power & Speed & \begin{tabular}{|l|} 
Bayesian Belief \\
network
\end{tabular} & NB & $\begin{array}{l}\text { Statistics } \\
\text { measures }\end{array}$ & $\begin{array}{l}\text { feature } \\
\text { extraction }\end{array}$ & $\begin{array}{l}\text { time scale } \\
\text { analisys }\end{array}$ & $\begin{array}{l}\text { envelope } \\
\text { analisys }\end{array}$ & CMeans & K Means & SVM & $\mathrm{Knn}$ & GBM & ARIMA & deep forest & CNN & ANN & DBN & DNN & RNN \\
\hline Jay Lee et ai [12] & & & & $x$ & $x$ & $x$ & & $x$ & $x$ & & & & & & & & & & & & & & & & & \\
\hline J. Yan et ai [13] & $x$ & $x$ & $x$ & & & & $x$ & & & & $x$ & $x$ & $x$ & $x$ & & & & & & & & & $x$ & & & \\
\hline Wo Jae Leea et ai [14] & & & $x$ & & & & $x$ & & & & $x$ & $x$ & & & & & $x$ & & & & & $x$ & $x$ & & & $x$ \\
\hline A. Susto, et ai [15] & & & 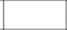 & $x$ & $x$ & $x$ & $x$ & $x$ & & & & & & & & & $x$ & $x$ & & & & & & & & \\
\hline N. Amruthnath et ai [16] & & & $x$ & & & & & & & & & & & & $x$ & $x$ & & & & & & & & & & \\
\hline S. Butte et ai $[17]$ & & & & & & & & & & & & & & & & & & & $x$ & & $x$ & $x$ & & $x$ & & \\
\hline A. Kanawaday et ai [18] & & & & & $x$ & & & & & $x$ & & & & & & & $x$ & & & $x$ & & & & & $x$ & \\
\hline
\end{tabular}

The choice of a given technique is not the only tedious task that researchers and engineers encounter to tackle the PdM problems. The choice of sensors also is not an easy task.

Table 1 summarize a list of papers and the variety of the Machine learning techniques and sensor used to finds a solution to the PdM problems

The distribution of the sensors and the technique of machine learning is explained in the figure 4 and figure 5 .

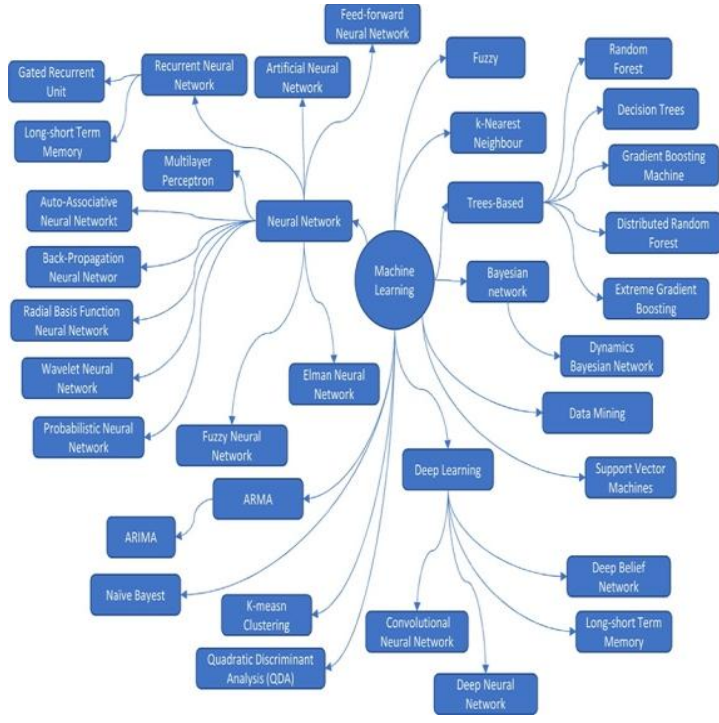

Fig. 3: Taxonomy of machine learning technique [11]

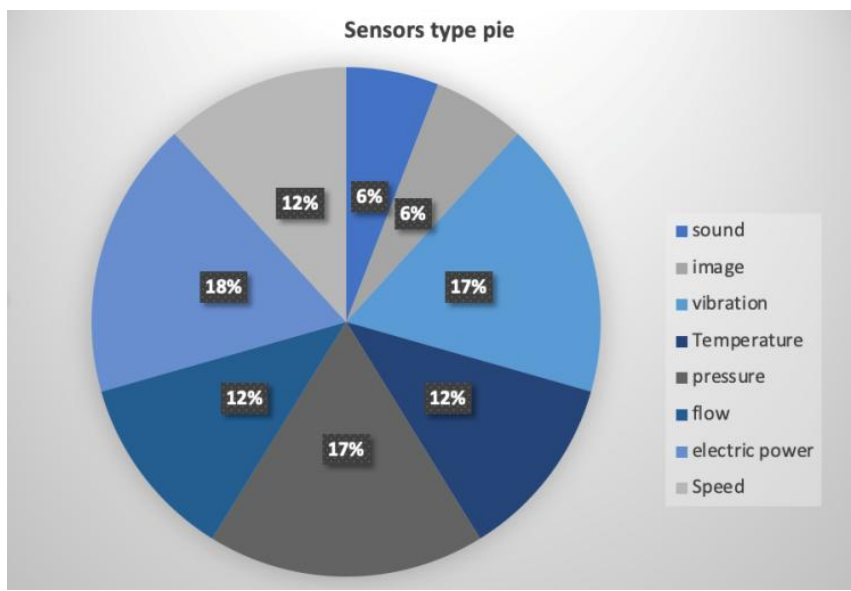

Fig. 4 Distribution of the Sensor types used in PdM.

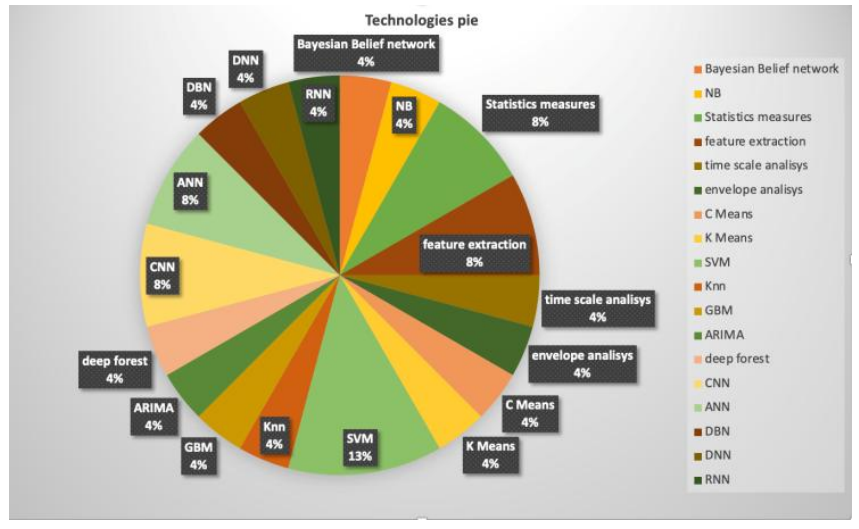

Fig. 5 distribution of the ML techniques used in PdM

Even though the sampling of article can seem small but the main challenge that we showcased here it that the researcher or engineer have to go through tedious task of experimenting a variety of sensors and also classification and prediction techniques, and here come in Smart Maintenance Simulator in the picture and for the only purpose of helping the researcher or engineer to speed the testing and experimenting of multiple techniques and try fast/false fast to remove the failing processes and focus on the one that gives the best results.

\section{PROPOSED MODEL}

This section sets forth a new Sensors Reference Model. Its purpose is to provide clear definitions and descriptions for sensors that can be used by our Smart Maintenance Simulator.

Today, there are a wide variety of sensors available for practically any industrial need. In PdM, these sensors offer real-time monitoring, including detection and reporting, as needed by a process. Data monitored and collected by sensors is sent for control and analysis, and any anomaly in a particular property is reported by emitting a notification by that system. This way, sensors enhance process efficiency and product quality, while ensuring that processes comply with best practices.

There are, in fact, many classifications for sensors [19]. Generally, this classification depends on the type of output signal or the physical, chemical or biological properties that they are measuring. However, other considerations should be taken for the architecture of our Smart Maintenance Simulator.

These considerations are regrouped in a new Sensors Reference Model named (SRM).

As shown on the figure 6 and figure 7, SRM is based on three classes of sensors and five layers to categorize each sensor. 


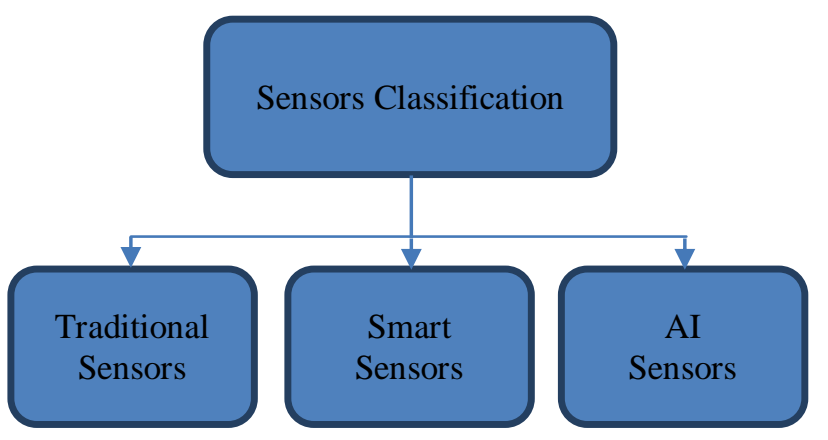

Fig. 6 Sensors classification.

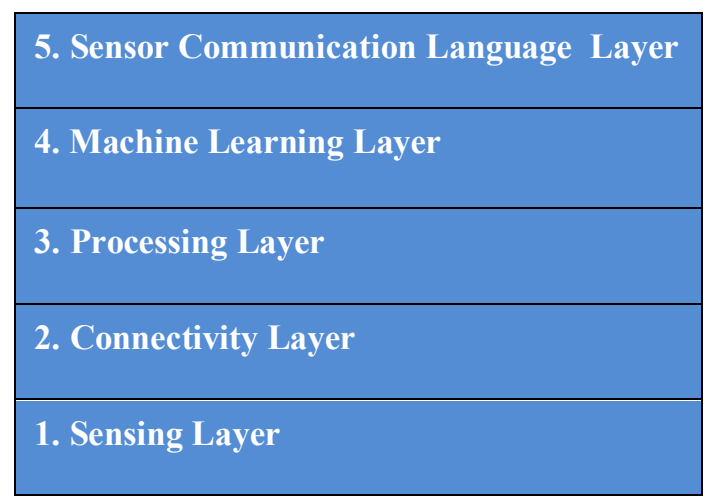

Fig. 7 Sensors Reference Model.

Level 1: Sensing Layer

The Sensors Reference Model starts with Level 1: Sensing layer is based on transducer devices that detect and convert in analog or digital signals, a wide variety of real world properties.

\section{Level 2: Connectivity Layer}

The most important function of Level 2 is the communication over network by the transmission and reception of data.

\section{Level 3: Processing Layer}

The role of Processing Layer is making quick or immediate decisions based on local data processing from the Sensing Layer.

\section{Level 4: Machine Learning Layer}

The most important function of Level 4 is to deploy the trained machine learning models, in order to make decisions and predictions based on new situation.

\section{Level 5: Sensor Communication Language Layer}

The Sensors Reference Model ends with Level 5: Sensor Communication Language Layer is based on higher-level language. For example, Natural language processing (NLP), voice command, voice synthesis, email notifications, SMS Alerts, etc. In this level, sensors would be able to communicate with humans using natural language.

According to our Sensors Reference Model, we can classify sensors in three classes:

\section{Class 1: Traditional Sensors}

A traditional sensor is a transducer device that would be able to:

- Detect a wide variety of real world physical, chemical or biological properties,

- Convert these properties in analog or digital signals
Basically, we classified in this category passive sensors, active sensors, analog sensors, digital sensors, etc.

\section{Class 2: Smart Sensors}

These Smart Sensors are built as IoT components that include Traditional Sensors (TS) and Information Technology (IT).

Generally, this class of Smart Sensors includes electronics, software, sensors, actuators, and network connectivity which would be able to:

- Exchange digital data with the Cloud Edge,

- Command and Control the actuators

- Communicate with other Machine (M2M)

Class 3: AI Sensors

This third class of sensors is built as an AI sensor that include Smart Sensors with Artificial Intelligence Technology. This category of sensors would be able to:

- $\quad$ Learn from collected data using Machine Learning and Deep learning algorithms.

- Deploy Machine Learning models

- Predict and monitor real time scenarios and take corrective actions in instant.

- Communicate with humans using natural language, notifications, emails, SMS Alerts, etc.

\section{SMART MAINTENANCE SIMULATOR}

According to an EY report prepared in collaboration with Oxford Analytica [20], smart sensors are the drivers of Industry 4.0 and the Internet of Things (IoT) in factories and workplaces. Once implemented at scale, the combination of sophisticated sensors and increased computational power will enable new ways to analyse data and gain actionable insights to improve many areas of operations. The result will be responsive and agile production processes that ensure and enhance performance across a range of industrial sectors.

Our hypothesis postulates that the impact of smart sensors and AI sensors on company performance, both today and in the up coming years, will be primarily through cost savings. How? with reducing failures and equipment downtime through predictive maintenance. In our opinion, smart sensors and AI sensors could play an important role in predictive maintenance.

To validate this hypothesis, we have developed a Smart maintenance Simulator called SmaSim based on our Sensors Reference Model. The architecture of SmaSim and its distributed sensors is shown in figure 8.

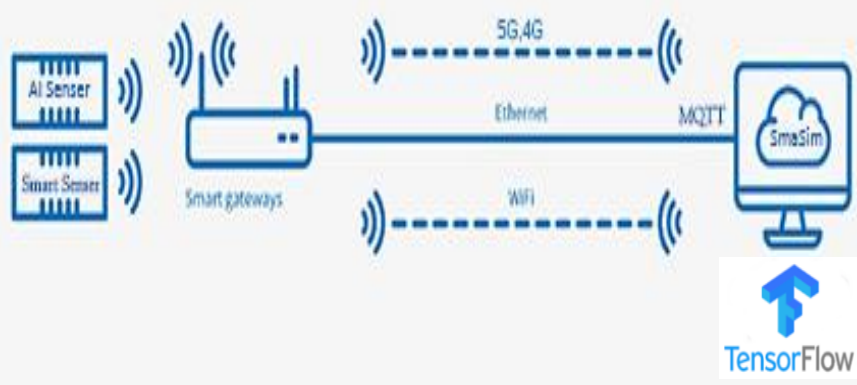

Fig. 8 Architecture of SmaSim and its distributed sensors

This Simulator is a TensorFlow python Framework deployed as a SaaS (Software as a Service) supporting experimentation with 
Sensors-oriented design. TensorFlow is a powerful data floworiented machine learning library created by the Brain Team of Google and made open source in 2015. It is designed to be easy to use and widely applicable on both numeric and neural networkoriented problems as well as other domains.

Basically, TensorFlow is a low-level toolkit for doing complicated math, and it targets researchers who know what they're doing to build experimental learning architectures to play around with them and to turn them into running software. It provides a suite of visualizing tools for example: As illustrated in figure 9, TensorBoard, that lets us to visualize the graphs and plot quantitative metrics about the graph with additional data.

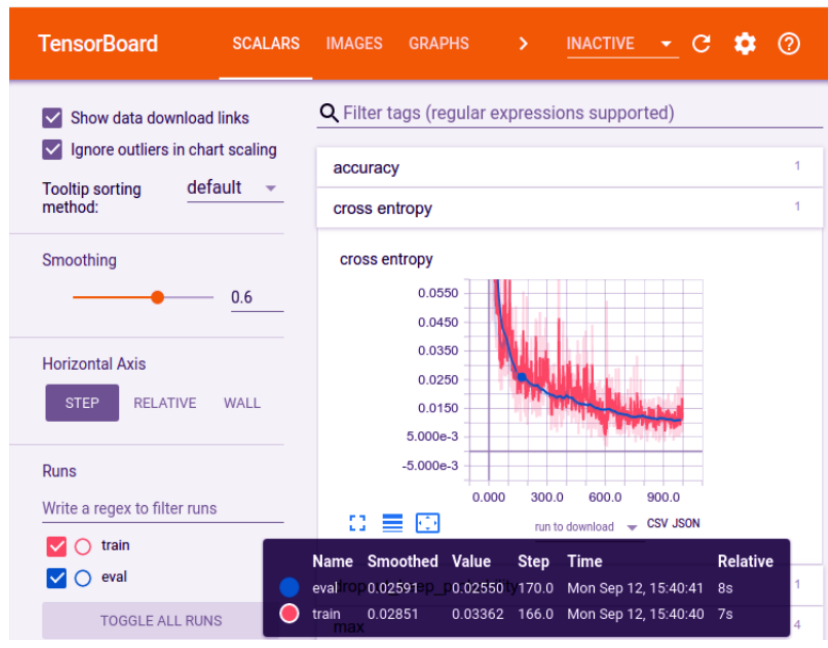

Fig. 9 Tracking and visualizing metrics with TensorBoard

This fully Smart environment was designed to simulate and deploy the topology of two types of distributed sensors. The first type of distributed sensors is the class 2 of our SRM named Smart Sensors and the second type of distributed sensors is the class 3 of our SRM named AI Sensors. These two types of distributed sensors can use quick response (QR) codes and wireless technology to enable communication between devices and SmaSim. The MQTT (message queue telemetry transport) protocol can be also used for the transfer of sensed data because it consumes very little bandwidth.

This process is called Sensors-Oriented Design Methodology, its consists of two modes:

1. The deployment mode: The main objective of this mode is, to establish a quick and easy sensors connection during the installation phase, with the help of the module's QR code: after the code is read, the distributed sensors will be connected to the SmaSim with Scan QR code shown in figure 10.
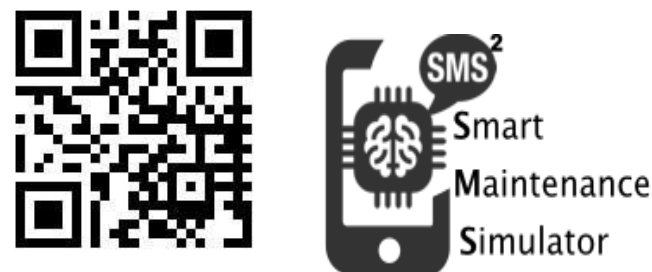

Fig.10 SmaSim deployment mode

2. The simulation mode: In this mode, the systems development process for Smart Sensor and AI Sensors applications in a smart factory requires preliminary research on the components and the interaction between them to achieve the proofs of concept and obtain verified prototypes. Modelling and simulation are alternative techniques that facilitate the analysis, design, and experimental testing of distributed sensors in SmaSim.

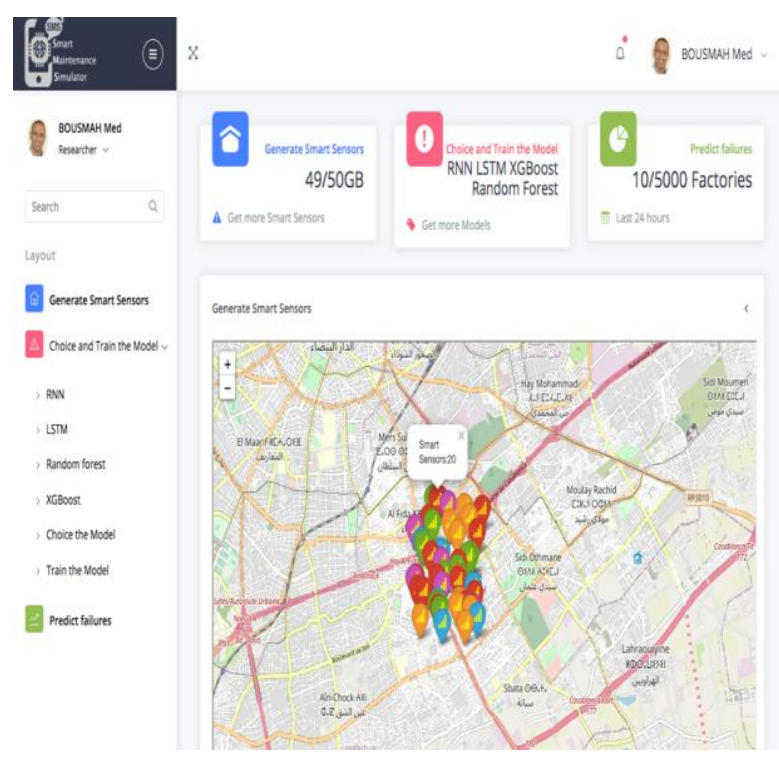

Fig.11 SmaSim Simulation Mode

As shown in figure 11, the SmaSim Simulation Mode is based on three steps:

- The first step is to select, configure and generate the advanced smart sensors (class 2 and class 3 of our SRM) using Sensors-oriented design methodology, the process of adding new sensors at any location or smart factories.

- The second step is to create, train and test the machine learning models. e.g. Decision tree, Random Forest, RNN, LSTM, etc. Machines are 'trained' to find rules, patterns and features from massive amounts of sensors data.

- The third step is to predict equipment failure from the real-time collected sensors data. The goal is to improve equipment condition, prolong the life of the equipment, and reduce the maintenance costs.

\section{CONCLUSION AND FUTURE WORK}

In this article, we have shown that machine learning can play an important role in predictive maintenance.

In this context, we have developed a Smart maintenance Simulator called SmaSim based on a new Sensors Reference Model named SRM which we have proposed. Its purpose is to provide clear definitions and descriptions for sensors that can be used by SmaSim in a new process called Sensors-Oriented Design Methodology.

The future work consists to experiment and validate SmaSim in real cases of PdM. The main goal is to know, at what level SmaSim can help researchers, engineers and practitioners to select appropriate Smart Sensors and Machine Learning algorithms for predictive maintenance in Smart Factories.

\section{REFERENCES}

[1] T. Wagner, C. Herrmann, S. Thiede, "Industry 4.0 Impacts on Lean Production Systems", Procedia CIRP 63 (2017), pp. 125-131 10.1016/j.procir.2017.02.041 
[2] J. Hull, " The second industrial revolution and the staples frontier in Canada: rethinking knowledge and history", Sci Can, 18 (1) (1994), pp. 22-37

[3] A. Toffler " The third wave", vol. 484, Bantam books, New York (1980).

[4] J. Bloem, M. Van Doorn, S. Duivestein, D. Excoffier, R. Maas, E. Van Ommeren, " The fourth industrial revolution: things to tighten the link between IT and OT contents", Groningen Sogeti VINT (2014).

[5] Mamoona Humayun, "Role of Emerging IoT Big Data and Cloud Computing for Real Time Application", (IJACSA) International Journal of Advanced Computer Science and Applications, Vol. 11, No. 4, 2020.

[6] H. M. Hashemian and W. C. Bean, "State-of-the-art predictive maintenance techniques", IEEE Transactions on Instrumentation and measurement, vol. 60 , no. 10 , pp. 34803492, 2011.

[7] M. Paolanti, L. Romeo, A. Felicetti, A. Mancini, E. Frontoni and J. Loncarski, "Machine Learning approach for Predictive Maintenance in Industry 4.0," 2018 14th IEEE/ASME International Conference on Mechatronic and Embedded Systems and Applications (MESA), Oulu, Finland, 2018, pp. 1-6, doi: 10.1109/MESA.2018.8449150.

[8] E. Frontoni, R. Pollini, P. Russo, P. Zingaretti and G. Cerri, "Hdomo: Smart sensor integration for an active and independent longevity of the elderly", Sensors, vol. 17, no. 11, pp. 2610, 2017.

[9] Carvalho, T.P.; Soares, F.A.; Vita, R.; Francisco, R.D.; Basto, J.P.; Alcalá, S.G. A systematic literature review of machine learning methods applied to predictive maintenance. Comput. Ind. Eng. 2019, 137, 106024.

[10] Jovani Dalzochioa, Rafael Kunsta,*, Edison Pignatonb, Alecio Binottoc, Srijnan Sanyalc, Jose Favillad, Jorge Barbosaa , "Machine learning and reasoning for predictive maintenance in Industry 4.0: Current status and challenges" Comput. Ind. 2020, 123, 103298

[11] Jovani Dalzochioa, Rafael Kunsta,*, Edison Pignatonb, Alecio Binottoc, Srijnan Sanyalc, Jose Favillad, Jorge Barbosaa , "Machine learning and reasoning for predictive maintenance in Industry 4.0: Current status and challenges" . Comput. Ind. 2020, 123, 103298- Fig. 9

[12] Jay Lee, Hung-AnKao, Shanhu Yang, "Service innovation and smart analytics for industry 4.0 and big data envirenement" Procedia CIRP 16 ( 2014 ) 3 -8

[13] J. Yan, Y. Meng, L. Lu and L. Li, "Industrial Big Data in an Industry 4.0 Environment: Challenges, Schemes, and Applications for Predictive Maintenance," in IEEE Access, vol. 5, pp. 23484-23491, 2017, doi: 10.1109/ACCESS.2017.2765544.

[14] Wo Jae Leea, Haiyue Wua, Huitaek Yunb, Hanjun Kimb, Martin B.G. Junb, John W. Sutherlanda "Predictive maintenance of machine tool systems using artificial intelligence techniques applied to machine condition data", Procedia CIRP 80 (2019) 506-511

[15] G. A. Susto, A. Schirru, S. Pampuri, S. McLoone and A. Beghi, "Machine Learning for Predictive Maintenance: A Multiple Classifier Approach," in IEEE Transactions on Industrial Informatics, vol. 11, no. 3, pp. 812-820, June 2015, doi: 10.1109/TII.2014.2349359.

[16] N. Amruthnath and T. Gupta, "A research study on unsupervised machine learning algorithms for early fault detection in predictive maintenance", 2018 5th International Conference on Industrial Engineering and Applications (ICIEA), 2018, pp. 355-361, doi: 10.1109/IEA.2018.8387124.

[17] S. Butte, A. R. Prashanth and S. Patil, "Machine Learning Based Predictive Maintenance Strategy: A Super Learning Approach with Deep Neural Networks," 2018 IEEE Workshop on Microelectronics and Electron Devices (WMED), 2018, pp. 1-5, doi: 10.1109/WMED.2018.8360836.

[18] A. Kanawaday and A. Sane, "Machine learning for predictive maintenance of industrial machines using IoT sensor data," 2017 8th IEEE International Conference on Software Engineering and Service Science (ICSESS), 2017, pp. 87-90, doi: 10.1109/ICSESS.2017.8342870.

[19] Kalsoom, T.; Ramzan, N.; Ahmed, S.; Ur-Rehman, M. "Advances in Sensor Technologies in the Era of Smart Factory and Industry 4.0", Sensors 2020, 20(23), 6783.

[20] EY, Oxford Analytica, "Sensors as drivers of Industry 4.0", An EY report prepared in collaboration with Oxford Analytica 